\title{
Collisional mechanics of the diagonal gaits of horses over a range of speeds
}

\author{
Sarah Jane Hobbs ${ }^{\text {Corresp., } 1}$, Hilary M Clayton ${ }^{2}$ \\ 1 University of Central Lancashire, Preston, United Kingdom \\ ${ }^{2}$ Sport Horse Science, Mason, Michigan, United States of America \\ Corresponding Author: Sarah Jane Hobbs \\ Email address: sjhobbs1@uclan.ac.uk
}

One of the goals of the neuromotor control system is to minimize the cost of locomotion by reducing mechanical energy losses. Collisional mechanics, which studies the redirection of the downwards motion of the center of mass (COM) by ground reaction forces (GRF) generated by the limbs, represents an important source of energy loss. The primary objective of this study was to compare collisional mechanics and the associated mechanical energy losses in horses performing diagonally-synchronized gaits over a range of speeds. It is to be expected that collisional energy losses will be high when the COM velocity vector is closely aligned with the GRF vector. This condition is achieved in piaffe, an artificial gait performed in dressage competitions that has a diagonal limb coordination pattern similar to trot but performed with little or no forward velocity. Therefore, we hypothesized that collisional energy losses would be higher in piaffe than in trot. Synchronized kinematic and GRF data were collected from three highly-trained horses performing piaffe, passage and trot at a range of speeds. Derived variables were vertical excursion and velocity of the trunk COM, fore and hind limb compression expressed as percentage reduction of standing limb lengths, range of limb pro-retraction, GRF vector magnitude and vector angle, collision angle $(\Phi)$, and mechanical cost of motion $\left(\mathrm{CoMot}_{\text {mech }}\right)$. Linear regression was used to investigate the relationship between CoMot $_{\text {mech }}$ and speed for each gait. Partial correlation was used to seek relationships between COM excursion and limb mechanics for each gait. Piaffe, passage and trot were clearly separated on the basis of speed. In all gaits the trunk was high at contact and lift off and descended to its lowest point in midstance following the pattern typical of spring mass mechanics. Mechanical cost was significantly $(p<.05)$ and inversely related to speed in trot and piaffe with the value increasing steeply as speed approached zero due to a near vertical orientation of both the COM velocity vector and the GRF vector. Limb compression during stance was significantly $(p<.05)$ linked to trunk COM vertical excursion in all gaits, with a stronger relationship in the forelimb. Hindlimb compression was, however, large in 
piaffe where the force magnitudes are notably smaller. The study illustrates the potential value of studying artificial gaits to provide data encompassing the entire range of locomotor capabilities. The results supported the experimental hypothesis by showing a threefold increase in collisional energy losses in piaffe compared with trot. In all gaits, dissociation between diagonal limb contacts and lift offs was thought to be an important strategy in reducing in collisional losses. Piaffe, the most costly gait, has similar characteristics to hopping on the spot. It appears that greater hindlimb compliance and a lower step frequency are important energy conservation strategies for piaffe. 
1 Collisional mechanics of the diagonal gaits of horses over a range of speeds 2

3 Sarah Jane Hobbs ${ }^{1}$ and Hilary M. Clayton ${ }^{2}$

$4 \quad{ }^{1}$ Centre for Applied Sport and Exercise Sciences, University of Central Lancashire, Preston, UK.

$5 \quad{ }^{2}$ Sport Horse Science, Mason, MI, USA.

6

7 Corresponding Author:

8 Sarah Jane Hobbs ${ }^{1}$,

9 Darwin Building DB201, Preston, Lancashire, PR1 2HE, UK.

$10 \quad$ Tel: +441772893328

11 E-mail: sjhobbs1@uclan.ac.uk

12 


\section{Abstract}

14 One of the goals of the neuromotor control system is to minimize the cost of locomotion by

15 reducing mechanical energy losses. Collisional mechanics, which studies the redirection of the

16 downwards motion of the center of mass (COM) by ground reaction forces (GRF) generated by

17 the limbs, represents an important source of energy loss. The primary objective of this study was

18 to compare collisional mechanics and the associated mechanical energy losses in horses

19 performing diagonally-synchronized gaits over a range of speeds. It is to be expected that

20 collisional energy losses will be high when the COM velocity vector is closely aligned with the

21 GRF vector. This condition is achieved in piaffe, an artificial gait performed in dressage

22 competitions that has a diagonal limb coordination pattern similar to trot but performed with

23 little or no forward velocity. Therefore, we hypothesized that collisional energy losses would be

24 higher in piaffe than in trot. Synchronized kinematic and GRF data were collected from three

25 highly-trained horses performing piaffe, passage and trot at a range of speeds. Derived variables

26 were vertical excursion and velocity of the trunk COM, fore and hind limb compression

27 expressed as percentage reduction of standing limb lengths, range of limb pro-retraction, GRF

28 vector magnitude and vector angle, collision angle $(\Phi)$, and mechanical cost of motion

$29\left(\mathrm{CoMot}_{\text {mech }}\right)$. Linear regression was used to investigate the relationship between CoMot $_{\text {mech }}$ and

30 speed for each gait. Partial correlation was used to seek relationships between COM excursion

31 and limb mechanics for each gait. Piaffe, passage and trot were clearly separated on the basis of

32 speed. In all gaits the trunk was high at contact and lift off and descended to its lowest point in

33 midstance following the pattern typical of spring mass mechanics. Mechanical cost was

34 significantly $(\mathrm{p}<.05)$ and inversely related to speed in trot and piaffe with the value increasing

35 steeply as speed approached zero due to a near vertical orientation of both the COM velocity 
36 vector and the GRF vector. Limb compression during stance was significantly $(\mathrm{p}<.05)$ linked to

37 trunk COM vertical excursion in all gaits, with a stronger relationship in the forelimb. Hindlimb

38 compression was, however, large in piaffe where the force magnitudes are notably smaller. The

39 study illustrates the potential value of studying artificial gaits to provide data encompassing the

40 entire range of locomotor capabilities. The results supported the experimental hypothesis by

41 showing a threefold increase in collisional energy losses in piaffe compared with trot. In all gaits,

42 dissociation between diagonal limb contacts and lift offs was thought to be an important strategy

43 in reducing in collisional losses. Piaffe, the most costly gait, has similar characteristics to

44 hopping on the spot. It appears that greater hindlimb compliance and a lower step frequency are

45 important energy conservation strategies for piaffe.

\section{Introduction}

47 Locomotion is the act of moving the body, represented by the center of mass (COM), from place

48 to place in relation to the environment. Quadrupeds achieve this by swinging the limbs back and

49 forth, alternating between stance and swing phases. During their stance phases the feet press

50 against the ground to develop ground reaction forces (GRF) that control movements of the COM

51 (Hobbs \& Clayton, 2013). The mechanical work is broadly divided into internal work used to

52 swing the limbs relative to the body and external work used to move the body, represented by the

53 COM, relative to the environment (Minetti et al., 1999). Movements of the limbs and body are

54 driven by muscular contractions that are fueled by the conversion of chemical energy into

55 mechanical energy. There is benefit to minimising energy expenditure for a given movement and

56 conversions between different types of mechanical energy fulfil this function (Cavagna Heglund

$57 \&$ Taylor, 1977). The mechanisms of inverted pendulum and spring mass mechanics are based

58 on exchanges between potential energy $\left(E_{p}\right)$, kinetic energy $\left(E_{k}\right)$ and elastic energy which are all 
59 types of mechanical energy. The exchanges occur cyclically during the stride to control the 60 vertical excursions of the COM (Geyer, Seyfarth \& Blickhan, 2006). Inverted pendulum

61 mechanics have been used to describe walking gaits, as the COM vaults over the grounded limb

62 during the stance phase, which allows energy exchanges between $E_{p}$ and $E_{k}$ to occur (Cavagna,

63 Heglund \& Taylor, 1977). In running gaits, the COM descends whilst the limb is grounded due

64 to limb compliance. This results in $E_{p}$ and $E_{k}$ being largely in phase, so instead energy is stored

65 in long elastic tendons as the COM descends then returned later in the step (Cavagna, Heglund \&

66 Taylor, 1977; Blickhan, 1989). Spring mass or spring-loaded inverted pendulum (SLIP) models

67 are often used to investigate running gaits (Ruina, Bertram \& Srinivasan, 2005). Energy

68 conservation by either inverted pendulum or spring mass mechanisms reduces the need for

69 muscular work to replenish lost mechanical energy.

70 During the stance phase of the stride, the motion of the COM is redirected from forward-

71 downward to forward-upward. Redirection of the COM contributes to the external work of

72 locomotion and is one of the main sources of mechanical energy losses (Bertram \& Hasaneini,

73 2013). Collisional mechanics describes the action of the limbs in redirecting the COM through

74 the generation of GRFs. The work done when a limb is in contact with the ground depends on

75 the angular relationship between the COM velocity vector $(\mathbf{V})$ and the resultant GRF vector $(\mathbf{F})$

76 (Lee et al., 2011). The collision angle is calculated as the difference between $\mathbf{F}$ and $\mathbf{V}$ shifted by

$77 \pi / 2$. Up to 0.3 radians the collision angle is closely related to the mechanical cost of motion (Lee

78 et al., 2011), which is a dimensionless metric of locomotor mechanical energy expenditure.

79 Considerable energy is expended when a horse's hooves push against the ground to redirect the 80 path of the COM, so collisions are a significant source of energy losses during locomotion

81 (Bertram \& Hasaneini, 2013). Limb coordination strategies that minimize the cost of reversing 
82 the motion of the COM from forward and downward at limb contact to forward and upward at

83 lift off are favored. One of the ways in which this is achieved is through the sequencing of limb

84 contacts with the ground, so that the COM velocity vector is gradually reoriented with each

85 successive limb collision (Ruina, Bertram \& Srinivasan, 2005). In this regard, the tölt with

86 individual footfalls has energetic advantages over the diagonally-synchronized footfalls of the

87 trot, as the work required to raise the COM in each stride is halved (Usherwood, 2019).

88 Each gait has a typical limb coordination pattern that may vary a little with speed and that

89 changes abruptly during transitions between gaits. Typically, horses walk at slow speeds, trot at

90 intermediate speeds and canter or gallop at fast speeds with each gait being used over a range of

91 speeds that allows the cost of transport to be maintained at a low level (Hoyt \& Taylor, 1981).

92 Transitions between gaits, which involve changes in limb coordination, may be triggered by the

93 need to reduce limb forces as speed increases (Farley \& Taylor, 1991). In walking and galloping,

94 each limb contacts the ground at a different time in the stride cycle which results in four

95 collisions per stride. Having a larger number of footfalls smooths the COM trajectory and

96 reduces collisional energy losses (Ruina, Bertram \& Srinivasan, 2005; Lee et al., 2011;

97 Usherwood, 2019). In diagonal gaits, such as trot, the synchronous diagonal contacts of fore and

98 hind limbs result in higher collisional energy losses and more abrupt changes in COM trajectory

99 compared with walk or gallop (Lee et al., 2011). Within a gait, limb sequencing can be adjusted

100 to reduce collisional energy losses. Adjustments of this nature occur in trotting horses, where

101 hind-first dissociation of the diagonal limb contacts has been associated with a significant

102 reduction in mechanical energy losses compared with fully synchronous contacts (Hobbs,

103 Bertram \& Clayton, 2016). 
104 In contrast to the parsimonious energetics of the natural gaits, the equestrian sport of dressage

105 rewards the horse for performing with great activity and impulsion, without regard for energetic

106 efficiency. The horse is trained to perform the natural gaits over an enhanced speed range and to

107 change gaits in response to a cue from the rider thus over-riding the horse's natural inclination to

108 make transitions based on physiological or biomechanical variables. Four types of trot are

109 performed that differ not only in speed but also in the body posture and limb kinematics. In order

110 of increasing speed $(m e a n \pm S D)$ they are collected trot $\left(3.20 \pm 0.28 \mathrm{~ms}^{-1}\right)$, working trot $(3.61 \pm 0.10$

$\left.111 \mathrm{~ms}^{-1}\right)$, medium trot $\left(4.47 \pm 0.23 \mathrm{~ms}^{-1}\right)$, and extended trot $\left(4.93 \pm 0.14 \mathrm{~ms}^{-1}\right)$ (Clayton, 1994). In

112 addition, highly trained dressage horses are taught to perform two, diagonally-coordinated

113 artificial gaits. Passage is a very slow, majestic trot performed at speeds in the range of 1.2-1.9

$114 \mathrm{~ms}^{-1}$ (Holmström Fredricson \& Drevemo, 1995; Weishaupt et al., 2009; Clayton and Hobbs,

115 2017). It is distinguished by the fact that the limbs appear to hover at their most elevated position

116 in the swing phase. Piaffe is performed with little or no forward movement with the limbs being

117 raised and lowered in diagonal pairs (Fédération Equestre Internationale, 2019). Passage and

118 piaffe represent the highest level of collection and self-carriage in competitive dressage.

119 This study investigates collisional mechanics in horses trained to maintain a diagonal limb

120 coordination pattern over a wide range of speeds that extend beyond those performed naturally in

121 order to investigate the spectrum of locomotor capabilities. The objective is to investigate

122 relationships between mechanical cost, COM excursion and spring-mass limb mechanics in

123 diagonally-coordinated equine gaits across a range of speeds. The hypothesis is that, as speed

124 decreases, the COM velocity will have a steeper vertical trajectory that will be associated with

125 higher mechanical energy cost. 
126 Methodology

127 This study was performed with approval from the institutional animal care and use committee

128 under protocol number 02/08-020-00 (Michigan State University, USA).

129 The subjects were three highly-trained dressage horses (mean $\pm \mathrm{SD}$, mass: $546 \pm 9 \mathrm{~kg}$ ) ridden by

130 the same highly experienced rider (mass $61.5 \mathrm{~kg}$ including saddle).

\section{Data Collection}

132 Data were collected as the horses moved along a $40 \mathrm{~m}$ by $12 \mathrm{~m}$ rubberized runway. A series of 133 four synchronized force plates were embedded in the middle part of the runway. The first and 134 last plates measured 60x120 cm (FP60120, Bertec Corp., Columbus, OH) and the middle two 135 measured 60x90 cm (FP6090, Bertec Corp., Columbus, OH). Force data were recorded at 960

$136 \mathrm{~Hz}$ per channel. Successful trials were judged subjectively by a dressage trainer as those in which

137 the horses moved straight and correctly for the gait being performed through the data collection 138 volume.

139 Retroreflective markers attached to the skin were tracked using 10 infra-red cameras (Eagle 140 cameras, Motion Analysis Corp., Santa Rosa, CA) recording at $120 \mathrm{~Hz}$ and motion analysis 141 software (Cortex 1.1.4.368, Motion Analysis Corp., Santa Rosa, CA). A full body kinematic 142 model as described in Hobbs, Richards \& Clayton (2014) was constructed with the omission of 143 trunk tracking markers T10 and T18, which provided a COM location for the trunk throughout

144 the dynamic trials. Markers located at the centre of rotation of the distal interphalangeal joints 145 were confirmed using radiographs.

146 Horses were familiarized with the research environment before commencing data collection and 147 warmed up appropriately. Trials were performed at a range of speeds in the diagonally- 
148 synchronized gaits of trot, passage and piaffe in a random order. The horses trotted at a range of

149 speeds to include trials at collected, working, medium and extended trot.

150 Data Processing and Analysis

151 All analyses were conducted using sagittal plane data.

152 The timings of hoof contacts and lift offs were identified from the force data using a threshold of

$15350 \mathrm{~N}$, except for piaffe where centre of pressure (COP) excursion was used to detect overlapping

154 limb contacts. Diagonal steps for the left forelimb and right hindlimb pair (LFRH) and the right

155 forelimb and left hindlimb pair (RFLH) were extracted when they were available.

156 Kinematic data were smoothed with a $4^{\text {th }}$ order Butterworth filter with $10 \mathrm{~Hz}$ cut off frequency

157 and kinetic data with a $4^{\text {th }}$ order Butterworth filter with $100 \mathrm{~Hz}$ cut off frequency. From these

158 data, selected variables were calculated for each frame of each available diagonal step. Trunk

159 COM location was extracted from the full body kinematic model and trunk velocity was then

160 calculated from the first derivative. Limb length and inclination were determined from the tuber

161 spinae scapulae (forelimb) and greater trochanter (hindlimb) to the centre of rotation of the distal

162 interphalangeal joint. Trunk COM vertical excursion was calculated as the difference between

163 minimum and maximum heights for each step. Limb compression, calculated as the difference in

164 vertical limb length during standing and minimum vertical limb length during the stance phase,

165 was normalized to a percentage change from the standing length. The range of limb pro-

166 retraction was calculated as the difference between the maximum protraction to maximum

167 retraction angles of the limb.

168 Vertical and longitudinal GRFs were summed over the fore- and hind limbs, and the resultant

169 force vector $\mathbf{F}$ was calculated from the summed components. Similarly, the resultant trunk 
170 velocity vector $\mathbf{V}$ was calculated from its vertical and longitudinal components. The

171 instantaneous collision angle $(\varphi)$ was calculated from the dot product of force on velocity and the

172 collision angle $(\Phi)$ was determined by force and velocity averaging over each step (Lee et al.,

173 2011). The mechanical cost of motion ( $\left.\mathrm{CoMot}_{\text {mech }}\right)$ was calculated for each step as the sum of the

174 dot product of force and velocity divided by the sum of absolute force multiplied by absolute

175 velocity (eqn. 1 from Lee et al., 2011).

$176 \operatorname{CoMot}_{\text {mech }}=\Sigma|\mathbf{F} \cdot \mathbf{V}| / \Sigma|\mathbf{F}||\mathbf{V}|$

177 To calculate summary vector variables (Hobbs, Robinson \& Clayton, 2018), GRF data were

178 normalized to horse mass. VecMag was calculated by vector summation of the individual vectors

179 divided by the number of samples contributing to the value. VecAng was determined

180 trigonometrically from the components of the vector magnitude and expressed relative to the

181 vertical with positive values being directed cranially. GRFs were down-sampled to $120 \mathrm{~Hz}$ and

182 GRF vector diagrams were constructed as examples of each gait.

183 As collision angles $>0.3 \mathrm{rad}$ were expected at slower speeds, linear regression was used to

184 investigate the relationship of $\mathrm{CoMot}_{\text {mech }}$ and speed across gait classifications. Partial correlation

185 controlling for horse was then used to examine the relationships between COM excursion and

186 limb mechanics for each gait classification separately. Significance was set at $\mathrm{p}<.05$.

\section{Results}

188 The range of gaits were classified by speed with no overlap between groups; piaffe $<0.6 \mathrm{~ms}^{-1}$, 189 passage 0.8 to $1.8 \mathrm{~ms}^{-1}$ and trot 2.0 to $5.12 \mathrm{~ms}^{-1}$. Metrics obtained for calculated variables 190 separated by gait classification and with trot data separated by speed are presented in Table 1. 


\section{COM}

192 For all gaits at all speeds the trunk COM followed the trajectory of a spring-mass model

193 associated with bounding gaits; the highest points of the diagonal stance phase were at contact

194 and lift off and the COM descended during the middle of stance (Figure 1). For piaffe, passage,

195 slow trot and fast trot, respectively, maximum height of the COM during each gait compared to

196 standing height was $18 \pm 15,42 \pm 21,5 \pm 17,-26 \pm 20 \mathrm{~mm}$ and minimum height compared to standing

197 height was $-51 \pm 22,-69 \pm 24,-71 \pm 15,-113 \pm 18 \mathrm{~mm}$. During trotting, the path of the COM became

198 longer and lower as speed increased.

199 Sagittal Plane GRF vectors

200 Vector diagrams representing the sagittal plane GRFs for the left and right diagonals in fast and

201 slow trot, passage, and piaffe (Figure 1D) show markedly lower vertical force magnitude,

202 represented by the height of the force vectors, in both fore and hind limbs for piaffe. The

203 horizontal spread of the force vectors represents the longitudinal forces. A wide spread of force

204 from braking to propulsion is evident in trot. Compared with trot, passage has higher magnitudes

205 of hind limb propulsive force and forelimb braking force and piaffe has a much smaller spread of

206 longitudinal force in both limbs. Force-time graphs representing mean vertical and longitudinal

207 forces for the RFLH diagonal step in each gait are shown in (Figure 1C). VecMag values and

208 vertical GRFs are considerably lower in piaffe compared with the other gaits (Table 1, Figure 1).

209 Collision angle

210 The instantaneous collision angle represents the deviation from a perpendicular relationship

211 between the COM velocity vector and GRF vector throughout stance (Figure 1B). In trot the

212 instantaneous collision angle falls and rises once during each diagonal step. The value falls to

213 approximately zero in the middle part of stance in trotting coinciding with the time when the

Peer) reviewing PDF | (2019:06:38360:1:0:NEW 14 Aug 2019) 
214 GRF vector and velocity vector are perpendicular. The first and second halves of the curve are

215 almost mirror images of each other. The shape of the instantaneous collision angle curves are

216 similar in passage and piaffe but the values in early and late stance get progressively higher. In

217 piaffe steps the fore and hindlimb contacts and lift offs overlap. During these periods of overlap

218 the vertical GRFs are small and there is a rapid decrease in the instantaneous collision angle as

219 the velocity and GRF vectors are closer to being orthogonal (Figure 1B). The collision angle $\Phi$

220 for $48 \%$ of the steps was $>0.3 \mathrm{rad}$. These steps were mainly found at piaffe and passage (Table

221 1).

222 Mechanical cost

223 The relationship between speed and mechanical cost for each gait is illustrated in Figure 2, where

224 significant relationships were found for piaffe $\left(\mathrm{R}^{2}=0.799, \mathrm{p}<.001\right)$ and $\operatorname{trot}\left(\mathrm{R}^{2}=0.333, \mathrm{p}<.001\right)$.

225 In piaffe, a small forward speed resulted in relatively low mechanical cost of motion but this

226 increased steeply as speed slowed to zero. Within passage, speed did not influence mechanical

227 cost significantly. During trotting, a slight but significant decrease in mechanical cost was found

228 with increasing speed.

229 Spring-mass mechanics

230 Relationships between trunk COM vertical excursion and limb mechanics varied with gait

231 classification (Table 2$)$. For all gaits, significant relationships $(\mathrm{p}<.05)$ were found between COM

232 vertical excursion variables and both fore and hind limb compression, with stronger and

233 consistently more significant $(\mathrm{p}<.01)$ relationships to the forelimb. Limb pro-retraction only

234 influenced COM excursion and height at trot, with greater pro-retraction of the forelimb or hind

235 limb the minimum height of the COM was lower. This was more pronounced in the hindlimb

$236(\mathrm{p}<.01)$ and as hindlimb pro-retraction increased, COM vertical excursion also increased $(\mathrm{p}<.01)$. 


\section{Discussion}

238 In this study the relationships between mechanical cost of motion, COM excursion and spring-

239 mass limb mechanics in diagonally-coordinated equine gaits were explored across a range of

240 speeds. The mechanical cost of motion was found to increase significantly with a decrease in

241 speed in trotting and piaffe, with the effect being most pronounced when forward speed was

242 close to zero. Overall, as speed decreased, the horizontal component of COM velocity reduced,

243 and as a consequence the velocity vector $\mathbf{V}$ became closer to the vertical. At the same time, limb

244 movements and the force vector $\mathbf{F}$ were directed more vertically. Convergence of the vectors $\mathbf{V}$

245 and $\mathbf{F}$ resulted in an increase in the mechanical cost of each collision with the ground.

246 Therefore, the hypothesis is supported.

247 Collisional mechanics provide an elegant method of comparing the cost of locomotion between

248 gaits and across species, since the values are dimensionless. As a diagonally coordinated gait, the

249 mechanical cost of trotting in dogs and goats is higher than walking and galloping, due to the

250 close sequencing of landing and lift-off of the fore and hindlimbs in each trotting step. Values

251 are reported to be 0.031 and 0.074 for walking, 0.212 and 0.192 for trotting, and 0.059 and 0.105

252 (rad) for galloping, for dogs and goats respectively (Lee \& Biewener 2011; Lee et al., 2011). In

253 this study, the collision angles at trot are higher than those reported for dogs and goats and

254 increase further for passage and piaffe. In addition to differences between species, the presence

255 of a rider may affect the movement patterns and energetics both through the gravitational and

256 inertial effects of the rider's weight and through the rider's effect on the horse's posture and

257 performance. Adding weight to the horse's back hollows the thoracolumbar spine (De Cocq et

258 al., 2004; 2009) and may change the weight distribution between the fore and hind limbs

259 (Schamhardt Merkens \& van Osch, 1991; Clayton et al., 1999; Licka Kapaun \& Peham, 2004), 
260 potentially altering limb loading and COM excursion. It is not known how the presence

261 compared to the absence of a rider affect mechanical energy exchanges.

262 The high mechanical cost in trotting can be alleviated somewhat by dissociation between fore 263 and hindlimb landings, with hind first landings reducing energy losses more than fore first 264 landings in horses (Hobbs, Bertram \& Clayton, 2016). The duration of hind-first dissociation 265 increases with trotting speed (Hobbs, Bertram \& Clayton, 2016), and a relatively long hind-first 266 dissociation is also a feature of passage (Holmström, Fredricson \& Drevemo, 1995; Clayton, 267 1997; Clayton \& Hobbs, 2017). In passage, any mechanical advantages gained by dissociation of 268 the diagonal limb contacts were likely overridden by the effects of the slower speed and 269 increased COM vertical excursion compared to trot. Greater energy losses are found in larger 270 down to up deflections of the COM (Bertram and Hasaneini, 2017). During forward-moving 271 trials of piaffe, although the speed was slower than passage the energy costs in the two gaits were 272 similar. Fore first landings are more commonly found in piaffe (Holmström, Fredricson \& 273 Drevemo, 1995; Clayton, 1997), but also in this study many of the steps had overlapping 274 contacts and lift offs. The similar costs for passage and forward-moving piaffe steps may be a 275 result of overlapping steps that potentially suggest a greater use of limb sequencing to minimize 276 cost and/or smaller down to up COM vertical excursions (Ruina, Bertram \& Srinivasan, 2005; 277 Usherwood, 2019). As speed tends towards zero in piaffe, energy is only required for upward 278 motion, the velocity and GRF vectors become more closely aligned, and the cost of motion rises 279 steeply. The rimless rolling wheel representing the collisional effects of successive footfalls 280 (Coleman, Chatterjee \& Ruina, 1997) no longer rolls and the limbs have a vertical trajectory.

281 Piaffe on the spot is possibly more akin to modelling a bouncing ball or a human hopping on the 282 spot (Blickhan, 1989), which may require different energy conservation strategies. 
283 During trotting $E_{k}$ and $E_{p}$ are largely in phase and so energy is conserved through elastic strain

284 energy storage in the spring elements of the limbs and trunk (Cavagna, Heglund \& Taylor,

285 1977). Any energy lost to the system, such as the losses during each collision must be replaced

286 by muscle work (Biewener, 2006). In piaffe, energy exchange is expected to be largely between

287 vertical $E_{k}, E_{p}$ and strain energy. Piaffe steps are slower than trotting steps (Holmström,

288 Fredricson \& Drevemo, 1995; Clayton, 1997), the shoulder and hock joints are continually more

289 flexed during the stance phase than in other diagonal gaits, and the fore and hind fetlock joints

290 extend less (Holmström, Fredricson \& Drevemo, 1995). The reduction in fetlock extension is

291 likely due to the lower vertical forces on the limbs (McGuigan \& Wilson, 2003), which is

292 illustrated by lower VecMag values here and probably explains why there is little change in

293 overall forelimb length during piaffe steps compared to standing length. The range of motion of

294 segments and joints during stance is also generally smaller in piaffe, which led Holmström,

295 Fredricson \& Drevemo (1995) to conclude that elastic strain energy may not be important.

296 Similar to piaffe, humans hopping on the spot maintain flexion of the limb joints, which

297 increases limb compliance and allows for greater energy storage if the hopping frequency is low

298 (Blickhan, 1989). In addition, most of the limb muscle work is isometric so changes in limb

299 length are mainly taken up by lengthening of the tendons (Blickhan, 1989). Our results illustrate

300 that COM vertical excursion is strongly affected by limb length changes, which suggests that

301 tendon lengthening and strain energy storage is a feature of piaffe, similar to hopping. This may

302 be more important in the hindlimb, as limb compliance is greater due to the greater change in

303 length compared to standing. If a large amount of the available mechanical energy is lost in

304 collisions with the ground when performing a stationary piaffe due to aligned GRF and velocity

305 vectors, reducing the step frequency and increasing hindlimb compliance to enhance energy 
306 storage in the tendons may be an important energy conservation strategy. In addition, isometric

307 and/or concentric muscle force production may be necessary to elevate the COM in each

308 successive step.

309 In contrast to the near-vertical limb orientation in piaffe, pro-retraction pendular motion,

310 particularly in the forelimb was important to COM vertical excursion in trot, together with spring

311 compression. The range of inverted pendulum motion increased with speed, indicating a longer

312 step length and the VecMag values are indicative of greater force production and consequently

313 greater limb spring compression (Biewener, 2006). These data conform well to the SLIP model

314 (Ruina, Bertram \& Srinivasan, 2005; Lee et al., 2011). In our study, the combination of increased

315 limb compression and greater pendular motion in trotting reduced the overall height of the COM

316 as speed increased, but with increased vertical COM excursion. A reduction in COM excursion

317 may be more efficient in human running, but not at the expense of greater limb work to smooth

318 the up-down motion (Bertram \& Hasaneini, 2013). The gradual reduction in cost of motion

319 reported here as trotting speed increased is expected to be the result of increased diagonal

320 dissociation (Hobbs, Bertram \& Clayton, 2016) and increased hindlimb pendulation with

321 increasing speed, which orients the GRF vector closer to the vertical during the absorbing phase.

322 Additional limb work may be required to achieve this (Bertram \& Hasaneini, 2013), so the net

323 energy in fast trot may be greater, but this is currently not known. In contrast, the role of the

324 forelimb in passage is to support the COM in a higher position at the start of stance which, in

325 effect, increases vertical COM excursion, as reported previously by Clayton \& Hobbs, (2017).

326 Since the forelimb acts principally as a passive limb spring (McGuigan \& Wilson, 2003), the

327 elevation appears to be achieved by limiting limb pro-retraction, which then influences weight

328 distribution between the fore and hind limbs (Clayton \& Hobbs, 2017). 
329 This study compared data from three Lusitano Grand Prix horses performing the three gaits of

330 trot, passage and piaffe. This limited our methods of statistical analysis and generalization to all

331 dressage horses performing these gaits should be made with caution.

332 Conclusions

333 This study has shown a negative relationship between velocity and collisional energy losses in

334 trot and piaffe. In general, a decrease in horizontal velocity of the trunk COM caused the velocity

335 vector to become more closely aligned with the GRF vector resulting in an increase in the

336 mechanical cost of each collision with the ground. This was particularly evident in piaffe, in

337 which, as speed approached zero, the COM vector and the GRF vector were both oriented almost

338 vertically throughout stance and collisional energy losses increased more than threefold

339 compared with steps that were moving forward at $0.6 \mathrm{~m} / \mathrm{s}$. Vertical excursions of the trunk COM

340 were strongly correlated to changes in limb spring lengths, which were more pronounced in the

341 forelimbs in all gaits. In piaffe, increasing hindlimb compliance is probably an important energy

342 conservation strategy.

\section{References}

344 Bertram, J.E.A. (2013). Gait as a solution, but what is the problem? Exploring cost, economy and

345 compromise in locomotion. Vet J 198, e3-e8; DOI: 10.1016/j.tvj1.2013.09.025

346 Bertram, J.E.A. \& Hasaneini, S.J. (2013). Neglected losses and key costs: tracking the energetics

347 of walking and running. J Exp Biol 216, 933-938; DOI:10.1242/jeb.078543

348 Biewener, A.A. (2006). Patterns of Mechanical Energy Change in Tetrapod Gait: Pendula,

349 Springs and Work. J Exp Zool 305A, 899-911; DOI: 10.1002/jez.a.334 
350 Blickhan, R. (1989). The spring-mass model for running and hopping. J Biomech 22, 11/12, $351 \quad 1217-27$.

352 Cavagna, G.A., Heglund, N.C. \& Taylor, C.R. (1977). Mechanical work in terrestrial 353 locomotion: two basic mechanisms for minimizing energy expenditure. Am J Physiol 233, 243354261.

355 Clayton, H.M. (1994). Comparison of the stride kinematics of the collected, working, medium 356 and extended trot in horses. Equine Vet J 26, 230-234.

357 Clayton, H.M. (1997). Classification of collected trot, passage and piaffe based on temporal 358 variables. Equine Vet J Suppl 23, 54-57.

359 Clayton, H.M. \& Hobbs, S.J. (2017). An exploration of strategies used by dressage horses to 360 control moments around the center of mass when performing passage. PeerJ 5, e3866;

361 DOI:10.7717/peerj.3866

362 Clayton, H.M., Lanovaz, J.L., Schamhardt, H.C. \& Wessum, R. van. (1999). Rider mass effects 363 on ground reaction forces and fetlock kinematics at the trot. Equine Vet J Suppl 30, 218-221.

364 Coleman, M.J., Chatterjee, A. \& Ruina, A. (1997). Motions of a rimless spoked wheel: a simple 365 three-dimensional system with impacts. Dynamics and Stability of Systems, 12:3, 139-159;

366 DOI: $10.1080 / 02681119708806242$

367 De Cocq, P., Clayton, H.M., Terada, K., Muller, M. \& van Leeuwen, J.L. (2009). Usability of 368 normal force distribution measurements to evaluate asymmetrical loading of the back of the 369 horse and different rider positions. Vet J 181, 266-273. 
370 De Cocq, P., van Weeren, P.R. \& Back, W. (2004). Effects of girth, saddle and weight on

371 movements of the horse. Equine Vet J 36, 758-763.

372 Farley, C.T. \& Taylor, C.R. (1991). A mechanical trigger for the trot-gallop transition in horses.

373 Sci 253, 306-308.

374 Fédération Equestre Internationale 2019. 25th edition.

375 https://inside.fei.org/sites/default/files/FEI_Dressage_Rules_2019_Clean_Version.pdf

376 Geyer, H., Seyfarth, A. \& Blickhan, R. (2006). Compliant leg behaviour explains basic dynamics

377 of walking and running. Proc R Soc B 273, 2861-2867; DOI:10.1098/rspb.2006.3637

378 Hobbs, S.J., Bertram, J.E.A. \& Clayton, H.M. (2016). The influence of diagonal dissociation and

379 speed on locomotor parameters in trotting horses. PeerJ 4, e2190; DOI: 10.7717/peerj.2190

380 Hobbs, S.J. \& Clayton, H.M. (2013). Sagittal plane ground reaction forces, centre of pressure

381 and centre of mass in trotting horses, Vet J 198, Suppl 1, e14-9; DOI: 10.1016/j.tvj1.2013.09.027.

382 Hobbs, S.J., Richards, J. \& Clayton, H.M. (2014). The effect of centre of mass location on 383 sagittal plane moments around the centre of mass in trotting horses. J Biomech 47, 1278-1286;

384 DOI: $10.1016 / j . j b i o m e c h .2014 .02 .024$

385 Hobbs, S.J., Robinson, M.A. \& Clayton, H.M. (2018). A simple method of limb force vector 386 analysis and its potential applications. Peer J 6, e4399; DOI: 10.7717/peerj.4399

387 Holmström, M., Fredricson, I. \& Drevemo, S. (1995). Biokinematic effects of collection on the 388 trotting gaits in the elite dressage horse. Equine Vet J 27, 281-287. 
389 Hoyt, D.F. \& Taylor, C.R. (1981). Gait and the energetics of locomotion in horses. Nature 292, $390 \quad 239-240$.

391 Lee, D.V. \& Biewener, A.A. (2011). BigDog-Inspired Studies in the Locomotion of Goats and 392 Dogs. Integ \& Comp Biol 51, 1, 190-202; DOI: 10.1093/icb/icr061

393 Lee, D.V., Bertram, J.E.A., Anttonen, J.T., Ros, I.G., Harris, S.L. \& Biewener, A.A. (2011). A 394 collisional perspective on quadrupedal gait dynamics. J R Soc Interface 8, 1480-1486;

395 DOI:10.1098/rsif.2011.0019

396 Licka, T., Kapaun, M. \& Peham, C. (2004). Influence of rider on lameness in trotting horses. 397 Equine Vet J 36, 734-736.

398 McGuigan, M.P. \& Wilson, A.M. (2003). The effect of gait and digital flexor muscle activation 399 on limb compliance in the forelimb of the horse Equus caballus. J Exp Biol 206, 1325-1336;

400 DOI:10.1242/jeb.00254

401 Minetti, A.E., Ardigò, L.P., Reinach, E. \& Saibene, F. (1999). The relationship between 402 mechanical work and energy expenditure of locomotion in horses. J Exp Biol 202, 2329-2338.

403 Ruina, A., Bertram, J.E.A. \& Srinivasan, M. (2005). A collisional model of the energetic cost of 404 support work qualitatively explains leg sequencing in walking and galloping, pseudo-elastic leg 405 behavior in running and the walk-to-run transition. J Theoret Biol 237, 170-192; DOI:

$406 \quad 10.1016 / j . j$ tbi.2005.04.004 
407 Schamhardt, H.C., Merkens, H.W. \& van Osch, G.J.V.M. (1991). Ground reaction force analysis 408 of horses ridden at walk and trot. In: Equine Exercise Physiology 3. Ed. S.G.B. Persson, A.

409 Lindholm and L.B. Jeffcott. ICEEP Publications, Davis, pp 120-127.

410 Usherwood, J.R. (2019). An extension to the collisional model of the energetic cost of support

411 qualitatively explains trotting and the trot-canter transition. J Exp Zool 2019, 1-11;

412 DOI:10.1002/jez.2268

413 Weishaupt, M.A., Byström, A., Von Peinen. K., Wiestner, T., Meyer, H., Waldern, N., Johnston,

414 C., Van Weeren, R. \& Roepstorff, L. (2009). Kinetics and kinematics of the passage. Equine Vet 415 J 41, 263-267. DOI 10.2746/042516409X397226. 


\section{Figure 1}

Data illustrating the mechanics of each gait taken from the RFLH diagonal (mean \pm standard deviation) (A-C), one RFLH step (D) and continuous RFLH-LFRH strides (E).

(A) Vertical excursion compared to standing $(\mathrm{mm})$ of the trunk COM (black), forelimb represented by scapula COM (blue) and hindlimb represented by the greater trochanter of the femur (red). Approximate limb inclinations are illustrated at footstrike, midstance and lift off. Forelimbs and hindlimbs are offset on the horizontal axis to position them cranially and caudally, respectively, from the COM. (B) Instantaneous collision angle (rad). (C) Forelimb (blue) and hind limb (red) ground reaction forces ( $N / \mathrm{kg}$ ). (D) Vector diagrams for the forelimbs (blue) and hindlimbs (red). (E) Footfall sequences and timing (s). 
Fast Trot

$$
\text { H. }
$$

A

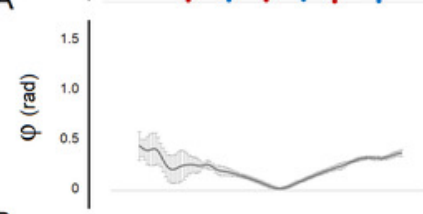

Slow Trot
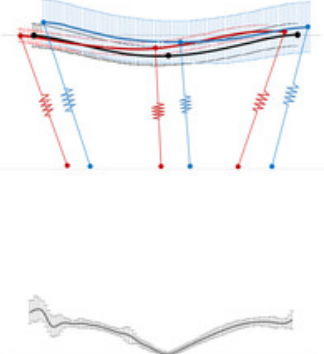

Passage
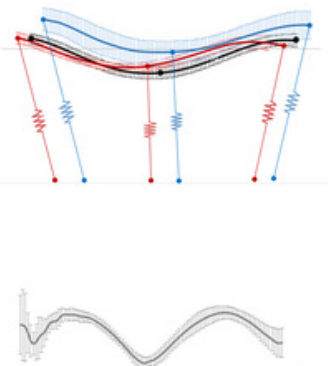
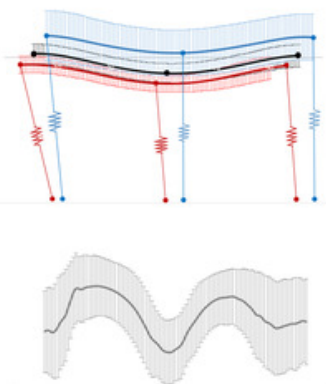

B
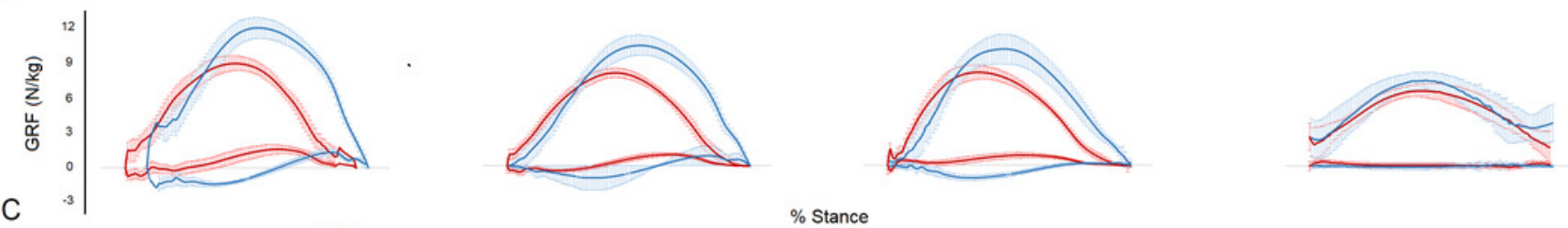

C
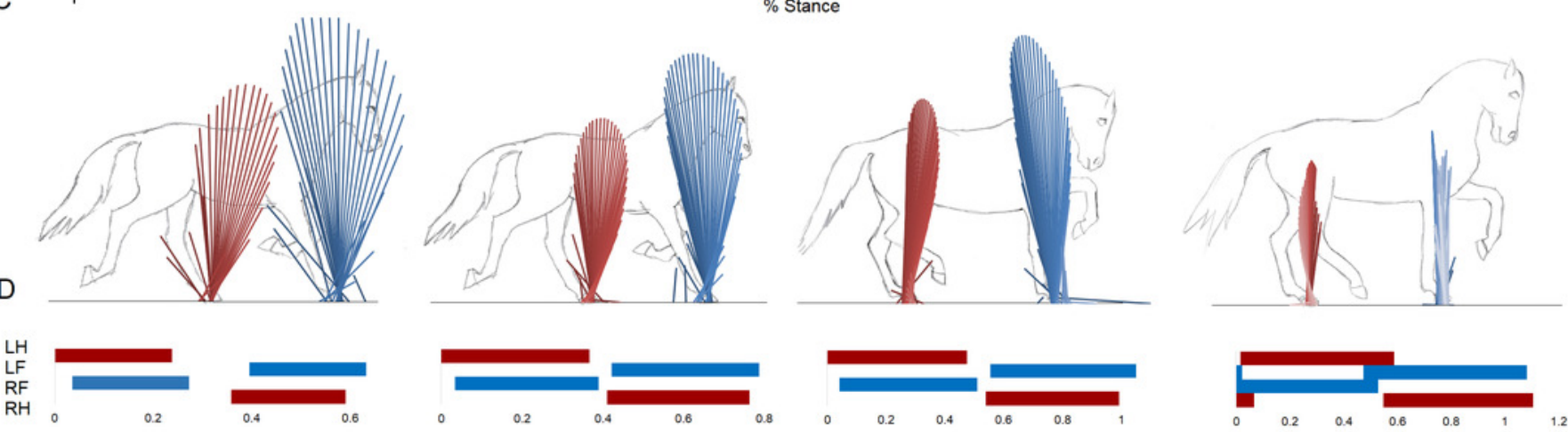

E

Time (s) 


\section{Table $\mathbf{1}$ (on next page)}

Measured variables for piaffe, passage, slow trot and fast trot.

Mean (s.d.) for collision angle $(\Phi)$, mechanical cost of motion $\left(\mathrm{CoMot}_{\text {mech }}\right)$, centre of mass vertical excursion (COMv excursion), limb mechanics ( $\Delta$ length $_{\mathrm{F}, \mathrm{H}} ;$ Pro-retraction $_{\mathrm{F}, \mathrm{H}}$ ) and summary vector variables (Mean vector magnitude in the forelimbs and hind limbs: $\operatorname{VecMag}_{\mathrm{F}, \mathrm{H}} ;$ Mean vector angle in the forelimbs and hind limbs: VecAng ${ }_{\mathrm{F}, \mathrm{H}}$ ) separated by gait classification. To illustrate the influence of speed in trotting, trot has also been separated into above and below $3.5 \mathrm{~ms}^{-1}$. 
1 Table 1: Measured variables for piaffe, passage, slow trot and fast trot.

2 Mean (s.d.) for collision angle $(\Phi)$, mechanical cost of motion $\left(\mathrm{CoMot}_{\text {mech }}\right)$, centre of mass

3 vertical excursion (COMv excursion), limb mechanics ( $\Delta$ length $_{\mathrm{F}, \mathrm{H}}$; Pro-retraction $\left.\mathrm{F}_{\mathrm{F}, \mathrm{H}}\right)$ and

4 summary vector variables (Mean vector magnitude in the forelimbs and hind limbs: $\operatorname{VecMag}_{\mathrm{F}, \mathrm{H}}$;

5 Mean vector angle in the forelimbs and hind limbs: VecAng $\mathrm{F}_{\mathrm{F}, \mathrm{H}}$ ) separated by gait classification.

6 To illustrate the influence of speed in trotting, trot has also been separated into above and below

$7 \quad 3.5 \mathrm{~ms}^{-1}$.

\begin{tabular}{|c|c|c|c|c|}
\hline & Piaffe & Passage & $\begin{array}{l}\text { Slow Trot } \\
<3.5 \mathrm{~ms}^{-1}\end{array}$ & $\begin{array}{l}\text { Fast Trot } \\
\geq 3.5 \mathrm{~ms}^{-1}\end{array}$ \\
\hline $\mathrm{n}$ & 65 & 26 & 73 & 24 \\
\hline Speed $\left(\mathrm{ms}^{-1}\right)$ & $0.19(0.16)$ & $1.27(0.19)$ & $2.68(0.39)$ & $4.37(0.57)$ \\
\hline Collision angle $\Phi(\mathrm{rad})$ & $0.88(0.33)$ & $0.38(0.04)$ & $0.27(0.02)$ & $0.26(0.01)$ \\
\hline CoMot $_{\text {mech }}$ & $0.73(0.21)$ & $0.34(0.03)$ & $0.21(0.02)$ & $0.19(0.01)$ \\
\hline $\mathrm{COM}_{\mathrm{v}}$ excursion $(\mathrm{mm})$ & $68.7(20.8)$ & $111.1(17.3)$ & $77.0(15.0)$ & $86.4(18.1)$ \\
\hline$\Delta$ length $_{\mathrm{F}}(\%)$ & $1.47(2.06)$ & $2.94(2.54)$ & $4.46(0.91)$ & $6.99(1.77)$ \\
\hline$\Delta$ length $_{\mathrm{H}}(\%)$ & $7.81(1.93)$ & $6.39(1.06)$ & $5.17(1.33)$ & $8.53(2.13)$ \\
\hline Pro-retraction $_{\mathrm{F}}(\mathrm{deg})$ & $5.1(3.1)$ & $22.3(4.7)$ & $33.1(5.0)$ & $39.5(4.7)$ \\
\hline Pro-retraction $_{\mathrm{H}}(\mathrm{deg})$ & $5.9(2.7)$ & $24.7(2.8)$ & $36.2(2.8)$ & $44.6(2.7)$ \\
\hline $\operatorname{VecMag}_{\mathrm{F}}(\mathrm{N} / \mathrm{kg})$ & $41.2(2.5)$ & $47.7(3.5)$ & $51.4(4.2)$ & $63.9(7.0)$ \\
\hline $\operatorname{VecMag}_{\mathrm{H}}(\mathrm{N} / \mathrm{kg})$ & $35.0(2.6)$ & $38.4(4.8)$ & $38.1(2.6)$ & $46.9(5.0)$ \\
\hline $\operatorname{VecAng}_{\mathrm{F}}(\mathrm{deg})$ & $0.12(1.61)$ & $-4.02(1.33)$ & $-1.59(1.35)$ & $-1.16(1.15)$ \\
\hline $\operatorname{Vec} \operatorname{Ang}_{H}(\mathrm{deg})$ & $0.37(2.20)$ & $3.72(2.86)$ & $2.84(1.62)$ & $6.06(2.41)$ \\
\hline
\end{tabular}




\section{Table 2 (on next page)}

Results of Partial correlation (controlling for horse) between COM vertical excursion and minimum height and limb mechanics. 
1 Table 2: Results of Partial correlation (controlling for horse) between COM vertical

2 excursion and minimum height and limb mechanics.

\begin{tabular}{|l|l|l|l|l|l|}
\hline & & $\Delta$ length $_{\mathrm{F}}$ & $\Delta$ length $_{\mathrm{H}}$ & Pro- & Pro- \\
& & & & retraction $_{\mathrm{F}}$ & retraction \\
$\mathrm{H}$
\end{tabular}

3

4 
Figure 2

Relationship between speed and mechanical cost of motion for each gait classification.

Key: piaffe $=$ blue, passage $=$ orange, trot $=$ grey .

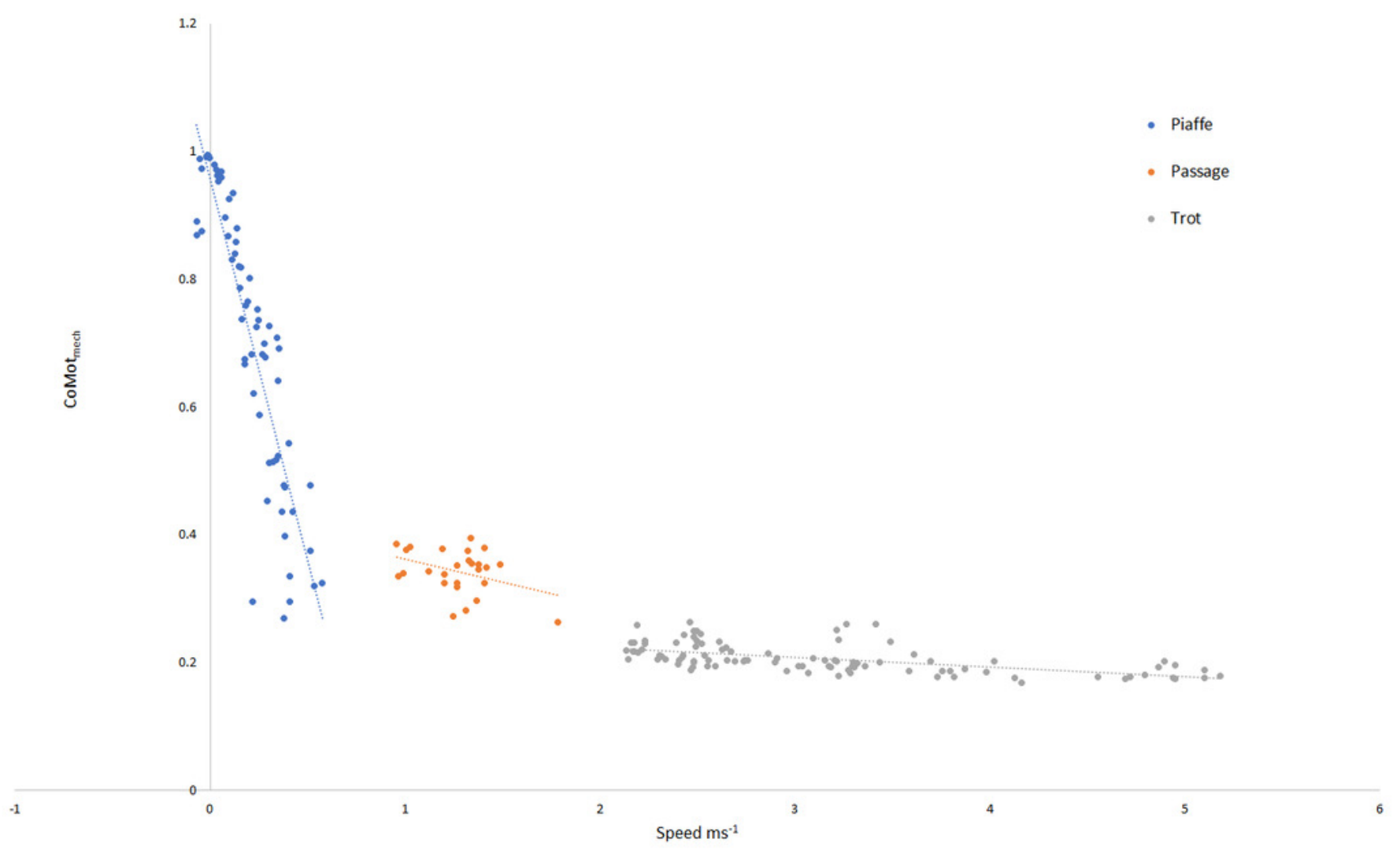

